\section{O OPEN ACCESS}

\title{
Individual-level and plant-level predictors of acute, traumatic occupational injuries in a manufacturing cohort
}

\author{
Kerry Souza, ${ }^{1}$ Linda F Cantley, ${ }^{2}$ Martin D Slade, ${ }^{2}$ Ellen A Eisen, ${ }^{3}$ David Christiani, ${ }^{1,4}$ \\ Mark R Cullen ${ }^{5}$
}

- Additional material is published online only. To view please visit the journal online (http://dx.doi.org/10.1136/ oemed-2013-101827).

1 Department of Epidemiology, Harvard School of Public Health, Boston, Massachusetts, USA

${ }^{2}$ Department of Occupational \& Environmental Medicine, Yale School of Medicine, New Haven, Connecticut, USA ${ }^{3}$ Department of Environmental Health Sciences, Berkeley School of Public Health, Berkeley, California, USA ${ }^{4}$ Departments of Environmental Health, Harvard School of Public Health, Boston, Massachusetts, USA ${ }^{5}$ Division of General Medical Disciplines, Stanford School of Medicine, Stanford, California, USA

\section{Correspondence to} Dr Mark Cullen, Stanford University School of Medicine, General Medical Disciplines, 1265 Welch Road, MSOB X-338, Stanford, CA 94305-5411, USA; mrcullen@stanford.edu

Received 3 September 2013 Revised 26 February 2014 Accepted 16 March 2014 Published Online First 12 April 2014

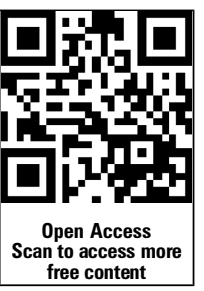

\section{ABSTRACT}

Objectives Workplace and contextual factors that may affect risk for worker injury are not well described. This study used results from an employee job satisfaction survey to construct aggregate indicators of the work environment and estimate the relative contribution of those factors to injury rates in a manufacturing cohort. Methods Principal components analysis was used to construct four plant-level factors from responses to a 32 question survey of the entire workforce, administered in 2006. Multilevel Poisson regression was used to evaluate the relationship between injury rate, individuallevel and plant-level risk factors, unionisation and plant type.

Results Plant-level 'work stress' (incident rate ratio $($ IRR $)=0.50,95 \% \mathrm{Cl} 0.28$ to 0.90 ) was significant in the multilevel model, indicating the rate of injury for an average individual in that plant was halved (conditional on plant) when job stress decreased by a tertile. 'Overall satisfaction', 'work environment' and 'perception of supervisor' showed the same trend but were not significant. Unionisation was protective (IRR $=0.40,95 \%$ $\mathrm{Cl} 0.17$ to 0.95 ) as was any plant type compared with smelter.

Conclusions We demonstrated utility of data from a human resources survey to construct indicators of the work environment. Our research suggests that aspects of the work environment, particularly work stress and unionisation, may have a significant effect on risk for occupational injury, emphasising the need for further multilevel studies. Our work would suggest monitoring of employee perceptions of job stress and the possible inclusion of stress management as a component of risk reduction programmes.

\section{INTRODUCTION}

Occupational injuries are a significant source of mortality, morbidity, disability and economic losses in the USA and worldwide. Concha-Barrientos ${ }^{1}$ estimated that occupational risk accounts for $8.1 \%$ of the global burden of morbidity and mortality (combined) from all unintentional injuries. In 2007, according to the Bureau of Labor Statistics, two million work-related injuries and illnesses (the vast majority of which are injuries) resulted in lost work time for workers in private industry. ${ }^{2}$ However, Leigh et al., taking into account widely recognised undercounting and underreporting of occupational injuries, estimated more than eight million non-fatal injuries actually occurred during

\section{What this paper adds}

- While risk factors for acute occupational injury related to job and demographic characteristics are increasingly well studied, less is known about the impact of workplace and contextual factors on injury risk. We used responses to an employee job satisfaction survey of 30163 employees at 56 locations of a single aluminium company under long-standing injury surveillance.

- Principal components analysis was used to construct plant-level factors from responses to the 32 question survey of the entire workforce administered in 2006. We used multilevel Poisson regression to evaluate the relationship between injury rate, individual-level factors and plant-level risk factors.

- Four dimensions around quality of work life emerged from the PCA: 'overall satisfaction with company', 'positive work environment', 'positive perception of supervisor' and 'job is stressful'.

- As average plant-level perceptions of job stress increased from poor to average or from average to good, the rate of injury for an average individual in that plant was halved, conditional on plant. Unionisation was also associated with lower risk (IRR=0.40, 95\% $\mathrm{Cl} 0.17$ to 0.95 ).

that time period. ${ }^{3}$ Physical job demands, worker characteristics such as age, sex, health, job experience and work hours are among the types of predictors that have been linked to risk for occupational injury. ${ }^{4-7}$ Others have reported on the roles of job strain ${ }^{8}$ and workplace factors such as safety climate, ${ }^{9}{ }^{10}$ training $^{11}$ and unionisation ${ }^{12}$ in different settings. A meta-analysis found that employee 'engagement' and job satisfaction were inversely and significantly correlated with lost workdays due to safety incidents. ${ }^{13} 14$ Studies of organisational 'climate' and particularly 'safety climate', concepts that refer to workers' shared perceptions of the culture of their workplace, have been linked to workplace injury experience. ${ }^{14}$ In the USA, manufacturing workplaces account for $17 \%$ of all injuries and illnesses, slightly higher than their $13 \%$ share of the workforce. ${ }^{2}$ This paper 
describes analyses of injury rates among production and maintenance employees working within 56 plants of a large aluminium manufacturing company that has locations distributed throughout the contiguous USA. Previous research in this cohort found associations between risk for occupational injury and worker characteristics such as age, sex, health, job experience and work hours, as well as physical job demand. ${ }^{4-7}$ During the study period, we observed great differences in injury rates across plant locations, including sixfold injury rate differences within plants of the same type. The observed differences in injury rate are consistent with previous research in this setting. ${ }^{15}$ These differences in injury experience were incompletely explained by compositional factors (ie, what kind of people work in the plant) or differences in occupation, assuming risk levels comparable to those previously reported. To help explain these differences, data on plant-level indicators of work environment were constructed from a questionnaire administered to all employees. These data were then incorporated into a multilevel regression model including established and suspect individual risk factors for acute, traumatic injury in this workforce, plant unionisation and plant type.

Like residents in communities and children in schools, worker cohorts and job groups can be viewed as nested within workplaces. In occupational health investigations, group-level covariates have most often been treated as fixed effects, though there are recent examples of multilevel analysis. ${ }^{16-18}$ Although some researchers have called for the treatment of job strain as a group-level variable, ${ }^{16} 1719$ this exposure is most often analysed as an individual-level variable. The first published study investigating job strain at the level of the workgroup (as a predictor of work disability) that accounted for correlated outcomes within workgroup was a 2009 report from the Finnish Public Sector Study. ${ }^{18}$ In this paper, we present results side-by-side from individual-level and multilevel regression models, each containing individual job-level and plant-level variables.

\section{METHODS}

\section{Cohort}

We examined injury and employment records for all production and maintenance workers at 56 plants, located throughout the USA, involved in the production of aluminium metal, aluminium products and related consumer products. Employment records $(n=47645)$ were obtained from the human resources (HR) data system for the time period from 1 October 2006 to 31 December 2007. Duplicate records $(n=2590)$ were deleted. Production and maintenance employees $(n=30506)$ at manufacturing locations were identified; rolled up hourly payroll data needed to create the underlying time scale for the Poisson regression models (ie, total number of hours worked) were available for 30163 workers, who made up the final eligible cohort. These data were derived from complete payroll for start and stop time (total number of hours in a shift), overtime, vacation and sick time. ${ }^{47}$

\section{Outcome data}

Incident acute, traumatic work-related injuries (injury) occurring between 1 October 2006 through 31 December 2007 was the outcome studied. The incident management system (IMS) system that captures detailed information on injuries is described by Pollack et al in previous work. ${ }^{20}$ Records of injury incidents were obtained from the company's real-time IMS. The IMS database, established in 1989 and modified over time, contains information on all reported work-related injuries that received any treatment including first aid, for all employees.
The IMS system is considered real time since incidents are recorded shortly after an incident occurs, depending on the level of severity.

Injury data are maintained by the company for purposes unrelated to this research and provided to the investigators under a research/service agreement in place since $1997 . .^{5}$ For this analysis, injuries were limited to instantaneous sprains, strains, burns, contusions, abrasions, fractions, lacerations, eye injuries, fractures, amputations, blisters, foreign bodies, punctures, bites and stings. Chronic injuries, sometimes classified as disease, particularly musculoskeletal disorders whose inception could not be traced to any one time or event were excluded.

\section{Employee satisfaction and organisational climate}

From 18 September to 6 October 2006, a company that offers HR research services administered a 32 question, employee job satisfaction survey to the study company's entire US workforce. The Yale research team provided input on survey questions, in particular recommending survey items from the CDC-NIOSH Quality of Work Life Module of the General Social Survey (http://www.cdc.gov/niosh/topics/stress/pdfs/QWLsurvey.pdf).

However, the wording of the final survey was determined by the HR research firm and their client. Employees were given paid work time to complete the survey in either an online or paper format. Overall response to the survey was $70 \%$ $(\mathrm{N}=29$ 247). Responses to questions 1-28 were provided on a five-point Likert scale of agreement (1='strongly disagree', $2=$ 'disagree', 3='neither agree nor disagree', 4='agree', $5=$ 'strongly agree').

Organisational climate (questions 29-32) was formatted with a frequency scale rather than a Likert scale: 1='always', 2='often', 3='sometimes', 4='rarely', 5='never'. For all questions, lower responses scores indicated more negative responses, that is, lower satisfaction, more stress, etc. The survey data set also contains the following demographic information associated with each record (one record per anonymous respondent): sex, minority (yes or no), years tenure at the company (categorical), function (operation/production, maintenance, administrative or professional), pay-type (hourly or salary, supervisory or non-supervisory), department within plant and geographic region. Because the survey was anonymous, responses were available for analysis only at the aggregate plant level.

\section{Exposure data}

Demographic and employment information were obtained from the employer's HR system. This data system contains fields for employee's sex, race, age, date of hire, hourly rate, W-2 income, plant, department and job title, job grade, and dates for all changes in job, job grade, compensation, disability leave, etc. Data obtained from the HR data system as of 1 October 2006 were used to create the following variables for regression modelling: sex (female, male), race (black, Hispanic, Asian, other), age (modelled in continuous 10-year groupings), time since hire (<1 year, 1-2 years, 2-3 years 3-5 years, 5-10 years and $>10$ years, as dummy variables comparing each category to $>10$ years) and job grade (ranging from 1 to 71 and modelled as continuous quintiles).

Employees were assigned age and time since hire as of 1 October 2006, and likewise compensation and job characteristics at the start of the study period were used. HR and IMS data were linked deterministically using encrypted personal identifiers.

The plant-level predictors included the workplace indicators derived from the survey, plant type and unionisation status of 
the hourly workers at each plant. Plant-level variables were constructed from survey responses first by using principal components analysis (PCA) to identify unique dimensions of response. We extracted components with an eigenvalue of 1.0 or higher. ${ }^{21}$ We identified four dimensions of response in the survey data set, which we named 'overall satisfaction', 'work environment', 'positive perception of supervisor' and 'job stress'. Questions included in each component are described in online supplementary table 1 . The components were then rotated orthogonally using varimax to maximise their independence. For ease of interpretability, the dimensions were modelled as scales rather than as linear equations. The scales and the dimensions performed similarly in the models, and the interpretation of each scale and its corresponding dimension is the same.

Fifty-six plants were categorised into eight categories, depending on plant type: smelting of raw aluminium; rolling of aluminium into product; production of consumer products (eg, foil); production of fastener systems or closure systems; production of engineered products; production of rigid packaging and metal-casting. Plant type of smelter was chosen as the referent because these plants involve the heaviest physical demand and have the highest injury rates. Plant unionisation status was coded as yes or no. All classifications of covariates were calculated in the same manner for all models.

\section{Sensitivity analysis: unavailable occupational demand}

We conducted an analysis to assess the potential sensitivity of effect estimates to confounding by a missing occupation variable. For this cohort, information on occupational demand is provided by a physical job demand survey (JDS) score. The physical demand required to perform a job was assessed by a single expert rater within each plant and rated 1 to 5 , sedentary to very heavy. JDS scoring had been conducted at six smelters by a health and safety manager trained by one of the authors (LC), but JDS scores were only available for 5322 workers employed during 2006-2007. We ran regression models with and without JDS scores within the data set of 5322 records and observed the effects on the estimates of sex, race/ethnicity, age, time since hire and job grade.

\section{Data analysis}

We used Poisson regression to model incident rate ratios (IRRs). All demographic and occupational characteristic variables that have been previously shown to be associated with occupational injury in data from this workforce and were included in analyses a priori. Random intercept log-linear models were fit to the injury data as Poisson-distributed, conditional on the plant variables. Model fit, following the addition of each predictor variable, was evaluated with pseudo-AIC criteria. Total hours worked during the study period was the offset variable (underlying person-time) for regression models.

The Poisson distribution fit the data adequately and the data were only slightly over-dispersed (ratio $\chi^{2} / \mathrm{df}=1.3$ ). As a sensitivity analysis, all regressions were repeated with negative binomials models. ${ }^{22}$ All risk ratios and their associated 95\% CI and $\mathrm{p}$ values were unchanged, so we report the results of Poisson models.

Four different models were constructed in order to find the optimal model to examine plant-level risk factors. Model 1 was an ordinary Poisson regression with individual-level factors only; model 2 incorporated a random effect for plant into model 1. Model 3 included both individual-level and plant-level variables but treated the latter as fixed effects in an individual- level model, and model 4, the full multilevel model, adds several plant-level risk factors.

These studies received Institutional Review Board approval for research involving human subjects from Yale University and the Harvard School of Public Health where the research was conducted. Analysis was conducted using SAS V.9.1.

\section{RESULTS}

The distribution of acute, traumatic injuries among the cohort is described in table 1 . In total, $91 \%$ of the cohort sustained no injuries over the 15 -month period, $7.5 \%$ sustained one injury, $1.3 \%$ two injuries and $0.3 \%$ three or more injuries. A higher proportion of men sustained injuries than women. There were statistically significant differences in proportion of workers sustaining an injury by race, age, time since hire, job grade, plant unionisation, plant type and by three of the four scales: overall satisfaction, perception of supervisor and job stress.

\section{Principal component analysis}

Four components (or dimensions) accounted for approximately $60 \%$ of the variability in the responses. The first component (eigenvalue $=14 / 32$ ) loaded heavily on feelings of satisfaction regarding the employer and was labelled 'overall satisfaction with company'. Component 2 (eigenvalue=2.5/32) was weighted towards questions about the job and the workplace climate and was labelled 'positive work environment'. The third component (eigenvalue $=2.2 / 32$ ) loaded heavily only on questions related to the respondents' supervisor and was therefore labelled 'positive perception of supervisor'. The final retained component (eigenvalue $=1.1 / 32$ ) loaded heavily only upon a set of four questions about psychological job demands and stress. This fourth component was labelled 'job is stressful'. Table 2 shows the Cronbach $\alpha$ scores for each component, and the distribution of plant-aggregated values of each of the four scales. Lower values indicate a more negative response. These four components were then included as plant-level covariates in models 3 and 4 described below.

\section{Models}

Results of the four different models of IRR are shown in table 3. The individual-level Poisson (model 1) suggests that Hispanic workers have an increased rate of injury (IRR $=1.27,95 \% \mathrm{CI}$ 1.08 to 1.49 ). Increasing age is protective (IRR $=0.93,95 \% \mathrm{CI}$ 0.88 to 0.99 ) and shorter time since hire increases risk (IRR range from 2.73 ( $<1$ year) to 1.21 (11-20 years) compared with $>20$ years). Here, there is little evidence of a relationship between sex and injury rate (IRR (Female) $=0.94$, 95\% CI 0.82 to 1.09). Compared with white workers, Asian workers appear to have lower injury rates (IRR $=0.68,95 \% \mathrm{CI} 0.47$ to 0.98$)$, and black race is not associated with injury rate (IRR $=1.05,95 \% \mathrm{CI}$ 0.89 to 1.20 ). Increasing job grade (in quintiles) is positively associated with injury (IRR=1.37, 95\% CI 1.31 to 1.43 ).

Model 2 contains the same terms as model 1, adding a random effects term for plant. This model allowed for variation in the intercept across plants in order to observe the differences in risk ratios and/or their $\mathrm{p}$ values. In this model, some substantial contributions to the outcome appear to be occurring at the plant level (variance for the random effect $=1.90, \mathrm{SE}=0.43$ ).

Model 2 indicates that women experience significantly higher injury rates than men (IRR $=1.44,95 \%$ CI 1.23 to 1.67$)$ after adjusting for race, age, time since hire and job grade. Correlations within plants were obscuring this relationship. Accounting for within-plant correlations also provides evidence that Asian workers do not have a lower rate of injury 
Table 1 Distribution of employees with acute, traumatic occupational injuries in a cohort of aluminium manufacturing workers ( $\mathrm{N}=30 \mathrm{163}$ )

\begin{tabular}{|c|c|c|c|c|c|}
\hline \multirow[b]{2}{*}{ Characteristic } & \multirow[b]{2}{*}{ Subjects in category } & \multicolumn{4}{|c|}{ Number of injuries (per cent) } \\
\hline & & Not injured & One injury & Two injuries & More than three injuries \\
\hline \multicolumn{6}{|l|}{$\mathrm{Sex}^{*}$} \\
\hline Male & 23301 & $21286(91.4)$ & $1674(7.2)$ & $274(1.2)$ & $67(0.3)$ \\
\hline Female & 6862 & $6146(89.6)$ & $578(8.4)$ & 109 (1.6) & $29(0.4)$ \\
\hline \multicolumn{6}{|l|}{ Race* $^{*}$} \\
\hline White & 20911 & $19040(91.1)$ & $1553(7.4)$ & $260(1.2)$ & $58(0.3)$ \\
\hline Black & 4420 & $4021(91.0)$ & $320(7.2)$ & $59(1.3)$ & $20(0.5)$ \\
\hline Hispanic & 3625 & $3257(89.9)$ & $299(8.3)$ & $54(1.5)$ & $15(0.4)$ \\
\hline Asian & 1002 & $937(93.5)$ & $61(6.1)$ & $3(0.3)$ & $1(0.1)$ \\
\hline Other & 205 & $177(86.3)$ & $19(9.3)$ & $7(3.4)$ & $2(1.0)$ \\
\hline \multicolumn{6}{|l|}{ Age $^{*}$} \\
\hline Under 25 & 1368 & $1226(89.6)$ & $113(8.3)$ & $25(1.8)$ & $4(0.3)$ \\
\hline 25 to 34 & 4862 & 4354 (89.6) & 409 (8.4) & $75(1.5)$ & $24(0.5)$ \\
\hline 35 to 44 & 7884 & $7165(90.9)$ & $598(7.6)$ & $92(1.2)$ & $29(0.4)$ \\
\hline 45 to 54 & 10261 & 9400 (91.6) & $706(6.9)$ & $124(1.2)$ & $31(0.3)$ \\
\hline Over 55 & 5788 & $5287(91.3)$ & $426(7.4)$ & $67(1.2)$ & $8(0.1)$ \\
\hline \multicolumn{6}{|l|}{ Time since hire (years)* } \\
\hline$<1$ & 5525 & $4893(88.6)$ & $488(8.8)$ & $117(2.1)$ & $27(0.5)$ \\
\hline $1-2$ & 2020 & $1805(89.4)$ & $182(9.0)$ & $24(1.2)$ & $9(0.5)$ \\
\hline $3-5$ & 2901 & 2645 (91.2) & $211(7.3)$ & $34(1.2)$ & $11(0.4)$ \\
\hline $6-10$ & 6076 & $5493(90.4)$ & $483(8.0)$ & $79(1.3)$ & $21(0.4)$ \\
\hline $11-20$ & 6177 & $5703(92.3)$ & $393(6.4)$ & $66(1.1)$ & $15(0.2)$ \\
\hline$>20$ & 7464 & $6893(92.4)$ & $495(6.6)$ & $63(0.8)$ & $13(0.2)$ \\
\hline \multicolumn{6}{|l|}{ Job grade* } \\
\hline $1-3$ & 5608 & $5229(93.2)$ & $332(5.9)$ & $36(0.6)$ & $11(0.2)$ \\
\hline $4-7$ & 6071 & $5556(91.5)$ & $420(6.9)$ & $74(1.2)$ & $21(0.4)$ \\
\hline $8-12$ & 6649 & 6078 (91.4) & $486(7.3)$ & $68(1.0)$ & $17(0.3)$ \\
\hline $13-21$ & 5686 & $5051(88.3)$ & $480(8.4)$ & $120(2.1)$ & $35(0.6)$ \\
\hline $22-71$ & 6149 & $5518(89.7)$ & $534(8.7)$ & $85(1.4)$ & $12(0.2)$ \\
\hline \multicolumn{6}{|l|}{ Unionised* } \\
\hline Yes & 14409 & $13299(92.3)$ & $872(6.1)$ & $186(1.3)$ & $52(0.4)$ \\
\hline No & 12100 & 11780 (97.4) & $301(2.5)$ & $16(0.1)$ & $3(0.02)$ \\
\hline \multicolumn{6}{|l|}{ Plant type* } \\
\hline Rigid packaging & 2580 & $2244(87.0)$ & $278(10.8)$ & $51(2.0)$ & $7(0.3)$ \\
\hline Casting & 6476 & $6360(98.2)$ & $112(1.7)$ & $3(0.05)$ & $1(0.02)$ \\
\hline Closure systems & 456 & 452 (99.3) & $2(0.44)$ & $2(0.22)$ & - \\
\hline Engineered products & 3730 & $3474(93.1)$ & $203(5.4)$ & $42(1.1)$ & $11(0.3)$ \\
\hline Fasteners & 2298 & $2271(98.8)$ & $25(1.1)$ & $2(0.1)$ & - \\
\hline Consumer products & 2700 & $2565(95.0)$ & $124(4.6)$ & $8(0.3)$ & $3(0.1)$ \\
\hline Rolled products & 2361 & $2314(98.0)$ & $37(1.6)$ & $7(0.3)$ & $3(0.1)$ \\
\hline Smelter & 2785 & $2346(84.2)$ & 325 (11.7) & $84(3.0)$ & $30(1.1)$ \\
\hline \multicolumn{6}{|l|}{ Satisfaction scalet } \\
\hline Low & 15309 & $14194(92.7)$ & $893(5.8)$ & $172(1.1)$ & $50(0.3)$ \\
\hline Medium & 8057 & 7803 (96.9) & $225(2.8)$ & $25(0.3)$ & $4(0.05)$ \\
\hline High & 3143 & $3082(98.1)$ & $55(1.8)$ & $5(0.2)$ & $1(0.03)$ \\
\hline \multicolumn{6}{|l|}{ Work environment scale } \\
\hline Poor environment & 17381 & $16352(94.1)$ & $838(4.8)$ & $149(0.9)$ & $42(0.2)$ \\
\hline Average environment & 7993 & 7607 (95.2) & $321(4.0)$ & $52(0.7)$ & $13(0.2)$ \\
\hline Good environment & 1135 & $1120(98.7)$ & $14(1.2)$ & $1(0.1)$ & - \\
\hline \multicolumn{6}{|l|}{ Supervisor scalet } \\
\hline Poor perception & 19204 & 17969 (93.6) & $1007(5.2)$ & $177(0.9)$ & $51(0.3)$ \\
\hline Average perception & 5950 & $5783(97.2)$ & $140(2.3)$ & $23(0.4)$ & $4(0.1)$ \\
\hline Good perception & 1355 & $1327(97.9)$ & $26(1.9)$ & $2(0.1)$ & - \\
\hline \multicolumn{6}{|l|}{ Stress scale* } \\
\hline High stress & 8395 & $7611(90.7)$ & $615(7.3)$ & $133(1.6)$ & $36(0.4)$ \\
\hline Medium stress & 10790 & $10315(95.6)$ & $398(3.7)$ & $61(0.6)$ & $16(0.2)$ \\
\hline Low stress & 7323 & $7153(97.7)$ & $160(2.2)$ & $8(0.1)$ & $2(0.04)$ \\
\hline
\end{tabular}

${ }^{*} p<0.05$.

†All employees in plant assigned average values, for descriptive purposes. 
Table 2 Distribution of survey responses in the cohort, by plant, $\mathrm{n}=56$

\begin{tabular}{llll}
\hline Scale & Mean (SD) & Range & Cronbach $\boldsymbol{\alpha}$ \\
\hline Satisfaction scale & $26.5(9.0)$ & $1-45$ & 0.94 \\
Work environment scale & $31.7(5.8)$ & $1-50$ & 0.90 \\
Supervisor scale & $19.6(6.3)$ & $2-30$ & 0.93 \\
Stress scale & $11.0(3.6)$ & $1-20$ & 0.80 \\
\hline
\end{tabular}

$(\mathrm{IRR}=0.83$, 95\% CI 0.55 to 1.25$)$. In model 2, age (in 10-year groupings) shows the same risk ratio, although no longer statistically significant (IRR $=0.92,95 \%$ CI 0.92 to 1.04 ).

All categories of time since hire are significant in model 2. Increasing job grade, which was associated with an increased injury rate in model 1 , has changed sign; model 2 indicates that a quintile increase in job grade is associated with a decrease in injury rate (IRR $=0.91 ; 95 \%$ CI 0.85 to 0.97 ).
Model 3 includes individual-level and plant-level predictors. In this model, women are at greater risk for injury than men $(\mathrm{IRR}=1.51,95 \%$ CI 1.31 to 1.75$)$. As in model 1 , Hispanic workers have higher injury risk (IRR $=1.45$, 95\% CI 1.22 to 1.71) than white workers. The estimates for black and Asian workers are near 1.0. Age was not significant (IRR $=1.02,95 \%$ CI 0.96 to 1.08 ). Time since hire remains a strong and significant predictor within all categories of tenure. Job grade is not significant in this model (IRR $=0.96,95 \%$ CI 0.91 to 1.02 ).

Results from this model indicate a strong association between plant-level variables and individual injury rate. Unionisation at the plant level appears to be protective $(\mathrm{IRR}=0.68,95 \% \mathrm{CI}$ 0.54 to 0.85 ). Plant type (referent $=$ smelter) is a strong predictor of injury. The estimates for all plant types are below 1, ranging from $I R R=0.03(95 \%$ CI 0.01 to 0.08$)$ for closure systems to $\mathrm{IRR}=0.70$ (95\% CI 0.60 to 0.80$)$ for rigid packaging. All four derived work environment scales are also significantly associated with lower risk. Plant-level increases of one tertile (ie, low to medium, or medium to high) in job satisfaction ( $I R R=0.68$, $95 \%$ CI 0.57 to 0.80 ), positive work environment (IRR $=0.82$,

Table 3 Individual-level and plant-level predictors of acute traumatic occupational injury in an aluminium manufacturing cohort: individual-level and multilevel models.

\begin{tabular}{|c|c|c|c|c|c|c|c|c|}
\hline \multirow[b]{2}{*}{ Variable } & \multicolumn{8}{|c|}{ Modelt } \\
\hline & \multicolumn{2}{|c|}{$\begin{array}{l}\text { Model 1: } \\
\text { individual-level model } \\
\text { ( } \mathrm{N}=22681 \text { workers) }\end{array}$} & \multicolumn{2}{|c|}{$\begin{array}{l}\text { Model 2: random } \\
\text { intercepts model } \\
\text { ( } N=22681 \text { workers, } \\
56 \text { plants) }\end{array}$} & \multicolumn{2}{|c|}{$\begin{array}{l}\text { Model 3: } \\
\text { individual-level model }\end{array}$} & \multicolumn{2}{|c|}{$\begin{array}{l}\text { Model 4: } \\
\text { random intercepts } \\
\text { model }\end{array}$} \\
\hline Female & 0.94 & (0.82 to 1.09 ) & 1.44 & $(1.23 \text { to } 1.67)^{* * *}$ & 1.51 & $(1.31 \text { to } 1.75)^{* * *}$ & 1.44 & $(1.23 \text { to } 1.67)^{* * *}$ \\
\hline Male & 1.0 & - & 1.0 & - & 1.0 & - & 1.0 & - \\
\hline \multicolumn{9}{|l|}{ Race } \\
\hline Black & 1.04 & (0.89 to 1.20$)$ & 1.10 & (0.94 to 1.31$)$ & 0.94 & (0.81 to 1.10$)$ & 1.07 & (0.91 to 1.27 ) \\
\hline Hispanic & 1.27 & $(1.08 \text { to } 1.49)^{* *}$ & 1.28 & $(1.06 \text { to } 1.54)^{\star *}$ & 1.45 & $(1.22 \text { to } 1.71)^{\star * *}$ & 1.30 & $(1.08 \text { to } 1.57)^{\star *}$ \\
\hline Asian & 0.68 & $(0.47 \text { to } 0.98)^{*}$ & 0.83 & (0.55 to 1.25 ) & 0.93 & (0.63 to 1.37 ) & 0.85 & (0.57 to 1.28$)$ \\
\hline White & 1.0 & - & 1.0 & - & 1.0 & - & 1.0 & - \\
\hline Age (10-year groupings) & 0.93 & $(0.88 \text { to } 0.99)^{*}$ & 0.98 & (0.92 to 1.04$)$ & 1.02 & (0.96 to 1.08$)$ & 0.99 & (0.93 to 1.05$)$ \\
\hline \multicolumn{9}{|l|}{ Time since hire (years) } \\
\hline$<1$ & 2.73 & $(2.28 \text { to } 3.28)^{* * *}$ & 2.41 & $(1.98 \text { to } 2.94)^{* * *}$ & 2.67 & $(2.20 \text { to } 3.25)^{* \star *}$ & 2.44 & $(2.00 \text { to } 2.97)^{* * *}$ \\
\hline $1-2$ & 1.18 & (0.91 to 1.53 ) & 2.00 & $(1.52 \text { to } 2.64)^{* * *}$ & 2.15 & $(1.64 \text { to } 2.83)^{* * *}$ & 2.08 & $(1.57 \text { to } 2.75)^{* * *}$ \\
\hline $3-5$ & 1.64 & $(1.32 \text { to } 2.04)^{* * *}$ & 1.60 & $(1.28 \text { to } 2.01)^{* * *}$ & 1.63 & $(1.28 \text { to } 2.00)^{* * *}$ & 1.64 & $(1.31 \text { to } 2.06)^{* * *}$ \\
\hline $6-10$ & 1.59 & $(1.34 \text { to } 1.88)^{* * *}$ & 1.63 & $(1.36 \text { to } 1.95)^{* * *}$ & 1.71 & $(1.43 \text { to } 2.05)^{* \star *}$ & 1.65 & $(1.38 \text { to } 1.98)^{* * *}$ \\
\hline $11-20$ & 1.21 & $(1.02 \text { to } 1.43)^{*}$ & 1.29 & $(1.08 \text { to } 1.53)^{\star *}$ & 1.22 & $(1.08 \text { to } 1.45)^{*}$ & 1.29 & $(1.08 \text { to } 1.54)^{* *}$ \\
\hline$>20$ & 1.0 & - & 1.0 & - & 1.0 & - & 1.0 & - \\
\hline Job grade (quintiles) & 1.37 & $(1.31 \text { to } 1.43)^{* * *}$ & 0.91 & $(0.85 \text { to } 0.97)^{* *}$ & 0.96 & (0.91 to 1.02 ) & 0.87 & $(0.81 \text { to } 0.94)^{* *}$ \\
\hline Unionised plant $(\mathrm{Y} / \mathrm{N})$ & & & & & 0.68 & $(0.54 \text { to } 0.85)^{* * *}$ & 0.40 & $(0.17 \text { to } 0.95)^{*}$ \\
\hline \multicolumn{9}{|l|}{ Plant type } \\
\hline Rigid packaging & & & & & 0.70 & $(0.60 \text { to } 0.80)^{* * *}$ & 0.87 & (0.19 to 3.92$)$ \\
\hline Casting & & & & & 0.09 & $(0.07 \text { to } 0.13)^{* * *}$ & 0.08 & $(0.03 \text { to } 0.22)^{* * *}$ \\
\hline Closure systems & & & & & 0.03 & $(0.01 \text { to } 0.08)^{* \star *}$ & 0.03 & $0.005,1.21 * * *$ \\
\hline Engineered products & & & & & 0.46 & $(0.38 \text { to } 0.54)^{* * *}$ & 0.23 & $(0.09 \text { to } 0.62)^{* *}$ \\
\hline Fastener & & & & & 0.05 & $(0.03 \text { to } 0.08)^{* * *}$ & 0.05 & $(0.01 \text { to } 0.19)^{* * *}$ \\
\hline Consumer products & & & & & 0.41 & $(0.33 \text { to } 1.51)^{* * *}$ & 0.44 & (0.15 to 1.25$)$ \\
\hline Rolled products & & & & & 0.04 & $(0.03 \text { to } 0.07)^{* * *}$ & 0.02 & $0.004,0.14^{* * *}$ \\
\hline Smelter & & & & & 1.0 & - & 1.0 & - \\
\hline Satisfaction scale (tertiles) & & & & & 0.68 & $(0.57 \text { to } 0.80)^{* * *}$ & 0.94 & (0.46 to 1.95$)$ \\
\hline Work environment scale (tertiles) & & & & & 0.82 & $(0.69 \text { to } 0.98)^{*}$ & 0.49 & (0.22 to 1.22$)$ \\
\hline Supervisor scale (tertiles) & & & & & 0.43 & $(0.37 \text { to } 0.50)^{* * *}$ & 0.70 & (0.25 to 2.02$)$ \\
\hline Stress scale (tertiles) & & & & & 0.60 & $(0.47 \text { to } 0.76)^{* * *}$ & 0.50 & $(0.28 \text { to } 0.90)^{*}$ \\
\hline
\end{tabular}

tResults are presented in the format of incidence rate ratio $(95 \% \mathrm{Cl})$.

${ }^{*} p<0.05 ;{ }^{* *} p<0.01 ;{ }^{* * *} p<0.001$. 
$95 \%$ CI 0.69 to 0.98 ), positive perception of the supervisor $(\mathrm{IRR}=0.43,95 \% \mathrm{CI} 0.37$ to 0.50$)$ and plant-level decreases in job stress (IRR $=0.60,95 \%$ CI 0.47 to 0.76$)$ are all associated with reductions in individual injury rate.

Model 4 is a multilevel Poisson model containing terms for all individual-level and plant-level variables and plant as a random effect. In contrast to model 3 , job grade is protective (IRR $=0.87,95 \%$ CI 0.81 to 0.94$)$. Apart from job grade, the largest differences between models 3 and 4 are observed with plant-level variables. Though the magnitude and direction of all associations remain similar, CIs widen for plant-level variables generally when modelled as random effects.

Unionisation is again associated with a lower individual injury rate $(\mathrm{IRR}=0.40,95 \% \mathrm{CI} 0.17$ to 0.95$)$. All plant types have a lower risk ratio compared with smelters, though for rigid packaging and consumer products, IRRs are no longer significant. Finally, though low work stress (IRR $=0.50,95 \%$ CI 0.28 to 0.90 ) and positive work environment (IRR $=0.49$, 95\% CI 0.22 to 1.22) still appear to be protective, the estimates for overall job satisfaction and perception of supervisor are attenuated and no longer significant. The risk ratios for all three of the four variables - the work environment, perception of supervisor and job stress-are relatively large. Interpretation of the IRR for stress is that as plant-level job stress decreases from high stress to medium stress or medium stress to low stress, the rate of injury for an average individual in that plant is halved, conditional on plant. The interpretation for the work environment variable would be nearly identical quantitatively, although this variable was not significant. As average plant-level perceptions of the work environment increase from poor to average or from average to good, the rate of injury for an individual in that plant is halved, conditional on plant.

When analyses stratified by injury severity are conducted (results not shown), the IRR for work environment (eg, higher score) and lower stress scores are both protective and significant within the set of first aid only injuries; only stress remains a protective factor against the less prevalent, more serious grades of injury.

\section{Sensitivity analysis}

In a subset of smelters, a rich job-level measure of overall physical demand from the JDS was available (data not shown). Adding JDS score did not change estimates of sex, race/ethnicity, age or job grade; however, the IRR for time since hire decreased among the newest hires by $7 \%$. This may reflect the concentration of jobs requiring high physical exertion among newer hires; $35 \%$ of the newest hires hold jobs rated as 'very heavy' compared with $4 \%$ of those employed for $1-2$ years, $11 \%$ of those employed 2-3 years, 2\% employed for 3-5 years, 2\% employed 5-10 years and 1\% employed more than 10 years (the relationship between tenure categories and JDS scores: Cramer's V correlation $\left.=0.24 ; \chi^{2}<0.0001\right)$.

\section{DISCUSSION}

Using multilevel modelling and a rich data source, we have shown that plant type, plant unionisation and perceptions of job stress are associated with individual rate of acute occupational injuries among employees distributed across 56 plant locations of an aluminium manufacturing company. We have also corroborated the findings of relationships of female sex, Hispanic race and short time since hire previously reported for subsets of this workforce. ${ }^{20}$ An additional variable, job grade, recently found to be associated with hypertension in a subset of this workforce, ${ }^{23}$ also appears to be a risk factor for acute injuries. Multilevel modelling of risk factors for acute, traumatic occupational injuries revealed relationships that were obscured by within-plant correlations; even when plant-level variables were absent. When both individual-level and plant-level variables were modelled accounting for the random effect of plant in a multilevel model, we saw adjustments to the estimates for plant-level predictors of individual injury rate that clarifies the contributions of both individual and plant level to individual injury rate compared with more traditional approaches to the data.

The analysis has several limitations. Confounding by unmeasured predictors of injury rate may be present in this data. A measure of specific job content/demand was not available for the whole cohort. Therefore, the sensitivity analysis, while reassuring, is limited to the subset of workers for whom physical job demand scores were available. It is possible that, in the larger workforce, the relationship between tenure and JDS score could be stronger or weaker than in the smelters, so the extent of confounding of the tenure effect could be more or less than that seen here.

Several other worker characteristics that have been associated with injury risk in this cohort, BMI/obesity, smoking status, education and overtime were missing for many in the workforce employed during this study period.

This analysis was conducted at two levels only-the level of the individual and the level of the plant within company. While workers at these plants are organised into at least one, and arguably more, level of organisation, department information was not available to us. We collapsed 56 plants into types according to the nature of the work done in the plant and modelled unionisation of the plants, with the hypothesis that stronger safety programming at unionised plants would result in lower injury rates. ${ }^{12} 24$ While we do not know if these plant-level variables are the most meaningful plant-level exposures in this workforce, by using available data from an existing survey, we have made some progress in parsing plants' contributions to individuals' injury rates.

\section{CONCLUSIONS}

Our measure of plant-wide job satisfaction includes indicators of the work environment that echo familiar occupational health constructs, namely, job demand, decision latitude and safety climate, from the results of a survey aimed at gauging employees' job satisfaction. ${ }^{25} 26$ While there is an abundant literature on the role of psychosocial factors in injury risk, especially for musculoskeletal injuries, ${ }^{10}{ }^{27-29}$ there is less in the literature for direct comparison of plant-level measures of these constructs with which to directly compare. Nonetheless, our findings do appear consistent with our priors and the broad perception that enhanced safety climate, worker satisfaction, supervisor support and less stress are conducive to lower organisation-wide injury rates and better outcomes. In particular, we find employee perceptions of work stress to be the most salient of these plant-level factors and the only one that remained significant in the final model.

The finding of a reduced risk ratio with unionisation in the multilevel model is also consistent with recent publications, although the issue remains unresolved. ${ }^{12}$ While there are many possible pathways by which such an association might result, one possible explanation is the association between union participation and a culture of safety. ${ }^{30}$

Our work highlights the value of multilevel modelling as a way to address location-level effects with less bias. It would also suggest monitoring of employee perceptions of job stress and the possible inclusion of stress management as a component of risk reduction programmes. 
Contributors All authors contributed to this work. KS designed the study, implemented all methods, helped to interpret the results, and participated in the drafting and editing of the paper at every stage. MRC posed the question, coordinated the research team and participated in all stages of study design as well as the writing and editing of the paper. LFC was responsible for classifying the physical demand of jobs, creating curating the survey data, data analysis and review of the final manuscript. MDS oversaw the management of the data, assisted with the analysis and provided feedback on the paper. EAE and DC assisted in study design, advised on the analysis methods and made substantive editorial contributions at all stages of manuscript preparation.

Funding This research was funded by two grants from the National Institute on Aging (Disease, Disability and Death in an Aging Workforce, NIH/NIA, 1 R01 AG026291-06 and Alcoa Inc.). The funders had no role in the design of this study; collection, management, analysis and interpretation of the data or the conduct of this study or preparation or approval of the manuscript. Alcoa reviewed the manuscript prior to publication.

Competing interests LFC, MDS and MRC receive a portion of their compensation at Yale and Stanford through a long-standing contractual agreement with Alcoa, Inc. The funders have no role in the design of this study; collection, management, analysis and interpretation of the data or the conduct of this study or preparation or approval of the manuscript.

Ethics approval Yale University and the Harvard School of Public Health.

Provenance and peer review Not commissioned; externally peer reviewed.

Data sharing statement As an alternative to providing a de-identified data set to the public domain, we allow access for the purpose of re-analyses or appropriate follow-on analyses by any qualified investigator willing to sign a contractual covenant with the host Institution limiting use of data to a specific agreed upon purpose and observing the same restrictions as are limited in our contract with Alcoa, such as 60-day manuscript review for compliance purposes.

Open Access This is an Open Access article distributed in accordance with the Creative Commons Attribution Non Commercial (CC BY-NC 3.0) license, which permits others to distribute, remix, adapt, build upon this work non-commercially, and license their derivative works on different terms, provided the original work is properly cited and the use is non-commercial. See: http://creativecommons.org/ licenses/by-nc/3.0/

\section{REFERENCES}

1 Concha-Barrientos M, Nelson DI, Fingerhut M, et al. The global burden due to occupational injury. Am J Ind Med 2005;48:470-81.

2 (BLS) BoLS. Non-fatal occupational injuries and illnesses requiring days away from work: United States Department of Labor 2011.

3 Leigh JP. Economic burden of occupational injury and illness in the United States. Milbank O 2011:89:728-72.

4 Vegso S, Cantley L, Slade M, et al. Extended work hours and risk of acute occupational injury: A case-crossover study of workers in manufacturing. Am I Ind Med 2007;50:597-603.

5 Pollack KM, Cheskin L. Obesity and workplace traumatic injury: does the science support the link? Inj Prev 2007;13:297-302.

6 Taiwo OA, Cantley LF, Slade MD, et al. Sex differences in injury patterns among workers in heavy manufacturing. Am J Epidemiol 2009;169:161-6.

7 Kubo J, Cullen MR, Cantley L, et al. Piecewise exponential models to assess the influence of job-specific experience on the hazard of acute injury for hourly factory workers. BMC Med Res Methodol 2013;13:89.

$8 \mathrm{Kim} \mathrm{HC}$, Min JY, Min KB, et al. Job strain and the risk for occupational injury in small- to medium-sized manufacturing enterprises: a prospective study of 1,209 Korean employees. Am J Ind Med 2009;52:322-30.
9 Clarke S. The relationship between safety climate and safety performance: A meta-analytic review. J Occup Health Psychol 2006;

11:315-27.

10 Smith TD, Dejoy DM. Occupational Injury in America: An analysis of risk factors using data from the General Social Survey (GSS). I Safety Res 2012; 43:67-74.

11 Robson LS, Stephenson CM, Schulte PA, et al. A systematic review of the effectiveness of occupational health and safety training. Scand I Work, Environ Health 2012;38:193-208.

12 Morantz AD. Coal Mine Safety: Do Unions Make a Difference? Industrial and Labor Relations Review, Forthcoming 2012:1-40.

13 Harter JK, Schmidt FL, Hayes TL. Business-unit-level relationship between employee satisfaction, employee engagement, and business outcomes: A meta-analysis. J Appl Psychol 2002;87:268-79.

14 Smith GS, Huang YH, Ho M, et al. The relationship between safety climate and injury rates across industries: The need to adjust for injury hazards. Accident Anal Prev 2006;38:556-62.

15 Clougherty JE, Souza K, Cullen MR. Work and its role in shaping the social gradient in health. Ann N Y Acad Sci 2010:1186:102-24.

16 Söderfeldt B, Söderfeldt $M$, Jones $K$, et al. Does organization matter? A multilevel analysis of the demand-control model applied to human services. Soc Sci Med 1997;44:527-34.

17 Elovainio M, Kivimäki M, Steen $\mathrm{N}$, et al. Job decision latitude, organizational justice and health: multilevel covariance structure analysis. Soc Sci Med 2004; 58:1659-69.

18 Laine $S$, Gimeno $D$, Virtanen $M$, et al. Job strain as a predictor of disability pension: the finnish public sector study. J Epidemiol Community Health 2009;63:24-30.

19 Leyland AH, Groenewegen PP. Multilevel modelling and public health policy. Scand J Public Health 2003;31:267-74.

20 Pollack KM, Agnew J, Slade MD, et al. Use of employer administrative Databases to identify systematic causes of injury in aluminum manufacturing. Am J Ind Med 2007;50:676-86.

21 Jolliffe I. Principal Component Analysis Encyclopedia of Statistics in Behavioral Science. John Wiley \& Sons, Ltd, 2005.

22 Rothman K, Greenland S, Lash T. Modern Epidemiology Third Edition. Philadelphia, PA: Lippincott Williams and Wilkins, 2008.

23 Clougherty JE, Eisen EA, Slade MD, et al. Workplace status and risk of hypertension among hourly and salaried aluminum manufacturing employees. Soc Sci Med 2009:68:304-13.

24 Samant $Y$, Parker D, Brosseau L, et al. Organizational characteristics of small metal-fabricating businesses in Minnesota. Int J Occup Environ Health 2007;13:160-6

25 Johnston JJ. Occupational injury and stress. J Occup Environ Med 1995;37:1199-203.

26 Swaen GM, van Amelsvoort LP, Bültmann U, et al. Psychosocial work characteristics as risk factors for being injured in an occupational accident. J Occup Environ Med 2004:46:521-7.

27 Lacey RJ, Lewis M, Sim J. Piecework, musculoskeletal pain and the impact of workplace psychosocial factors. Occup Med 2007:57:430-7.

28 Sobeih TM, Salem O, Daraiseh N, et al. Psychosocial factors and musculoskeletal disorders in the construction industry: a systematic review. Theor Issues Ergon Sci 2006:7:329-44.

29 Simon M, Tackenberg P, Nienhaus A, et al. Back or neck-pain-related disability of nursing staff in hospitals, nursing homes and home care in seven countries-results from the European NEXT-Study. Int I Nurs Stud 2008;45:24-34.

30 Gillen M, Baltz D, Gassel M, et al. Perceived safety climate, job demands, and coworker support among union and nonunion injured construction workers. I Safety Res 2002:33:33-51. 